\title{
Posterior-only surgical correction with heavy halo-femoral traction for the treatment of rigid congenital scoliosis associated with split cord malformation
}

Hong-Qi Zhang, Ang Deng ${ }^{*}$ D, Ming-Xing Tang, Shao-Hua Liu, Yu-Xiang Wang and Qi-Le Gao

\begin{abstract}
Background: Whether or not, prophylactic neurosurgical interventions of split cord malformation (SCM) before undertaking corrective surgery was the focus of debate. The present study was performed to evaluate the safety and efficacy of posterior-only surgical correction with heavy halo-femoral traction for the treatment of rigid congenital scoliosis (RCS) associated with SCM.
\end{abstract}

Methods: From 2011 to 2017, 24 patients suffered from RCS associated with SCM underwent posterior-only surgical correction with heavy halo-femoral traction. The apex of the deformity was lumbar $(n=9)$, thoracic $(n=11)$, and thoracolumbar $(n=4)$. There were 13 cases of failure of segmentation; 4 cases of failure of formation and 7 cases of mixed defects. Based on SCM classification, there were 14 patients with SCM type 1 and 10 patients with SCM type 2. The Scoliosis Research Society (SRS)-22 and modified Japanese Orthopaedic Association (mJOA) scores were assessed preoperatively and at the final follow up.

Results: The mean duration of surgery was $327.08 \pm 43.99$ min and the mean blood loss was $1303.33 \pm 526.86 \mathrm{ml}$. The mean follow-up period was $20.75 \pm 8.29$ months. The preoperative mean coronal Cobb angle was $80.38^{\circ} \pm$ $13.55^{\circ}$; on the bending radiograph of the convex side, the mean Cobb angle was $68.91^{\circ} \pm 15.48^{\circ}$; the mean flexibility was $15.04 \% \pm 7.11 \%$. After heavy halo-femoral traction, the mean coronal Cobb angle was reduced to $56.89^{\circ} \pm 13.39^{\circ}$. After posterior-only surgical correction, postoperative mean coronal Cobb angle was further reduced to $32.54^{\circ} \pm 11.33^{\circ}$. The postoperative mean correction rate was $60.51 \% \pm 7.79 \%$. At the final follow up, the corrective loss rate of Cobb angle was only $3.17 \%$. The SRS-22 total score improved at the final follow-up evaluation compared with the preoperative SRS-22 total score. The spinal cord function was stable and there were no new neurological symptoms after correction. There were no significant differences between final follow-up and preoperative mJOA total scores.

Conclusions: Without prophylactic neurosurgical intervention and spine-shortening osteotomy, posterior-only surgical correction with heavy halo-femoral traction could be safe and effective for the treatment of RCS associated with SCM.

Keywords: Split cord malformation, Halo-femoral traction, Posterior-only, Rigid congenital scoliosis

\footnotetext{
* Correspondence: deng.aimar@163.com

Department of Spine Surgery, Xiangya Hospital of Central South University,

Xiangya Road 87, ChangSha 410008, China
}

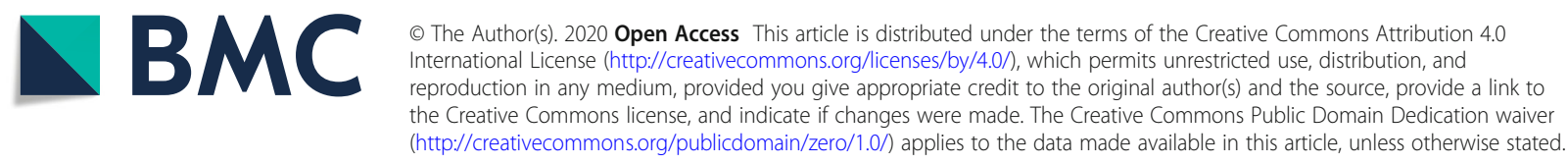




\section{Background}

Split cord malformation (SCM) is a rare type of congenital spinal cord abnormality however, it might be a common finding associated with congenital scoliosis (CS). Pang's new nomenclature was established to avoid confusion between diplomyelia and diastematomyelia. In Type I SCM, the spinal cord is divided into two halves with two different dural sleeves by a fibrocartilaginous or bony spur. In Type II SCM, there is only a common intradural fibrous band and dural sac without bony spur $[1,2]$. The treatment of CS associated with SCM is complex, and the spinal cord abnormality and spinal deformity must be taken into account. Prophylactic neurosurgical intervention of SCM before undertaking corrective surgery is the focus of debate in spine surgery and neurosurgery [3-5]. Conventional approach for management of CS associated with SCM was first to perform neurosurgical intervention for SCM and then to perform correction of scoliosis 3 to 6 months later. To date, there are no studies concerning the application of posterior-only surgical correction with heavy halofemoral traction for the treatment of rigid congenital scoliosis (RCS) associated with SCM in the absence of prophylactic neurosurgical intervention and spineshortening osteotomy. Therefore, the present study was performed to evaluate the safety and efficacy of posterior-only surgical correction with heavy halofemoral traction for the treatment of RCS associated with SCM retrospectively.

\section{Methods}

\section{General data}

From 2011 to 2017, 24 patients (9 males and 15 females; age, $10-24$ years; average age, $16.38 \pm 4.56$ years), suffered from RCS associated with SCM, were treated at our Department. In all patients X-ray, CT and MRI examinations revealed: the apex of the deformity was lumbar $(n=9)$, thoracic $(n=11)$, and thoracolumbar $(n=4)$; based on CS classification, all patients were divided into 3 types, including 13 cases of failure of segmentation, 4 cases of failure of formation, and 7 cases of mixed defects; based on SCM classification, all patients were divided into 2 types, including 14 cases of SCM type 1 and 10 cases of SCM type 2; only 7 patients had complication of syringomyelia. Thorough neurological examination, including muscle strength, sensation, pathological and physiological reflexes, was carried out in all of the patients. No apparent neurologic dysfunction was found before surgery. Only 1 patient complained of irregular urination, and thus underwent additional neural electromyography and urodynamic test. Based on all normal results of tests, this patient was also included in this study. The Ethics Committee of Xiangya Hospital of Central South University approved the study. All methods were performed in accordance with the relevant guidelines and regulations. Informed consent for study participation was obtained.

The indications for posterior-only surgical correction with heavy halo-femoral traction were based on the following criteria; (1) the patients suffered from CS associated with SCM; (2) coronal Cobb angle more than $60^{\circ}$; (3) the flexibility less than $30 \%$; (4) consideration of the progression of the scoliosis.

Excluded criteria were based on; (1) SCM-related neurological symptoms were found before surgery, or neurological symptoms occurred during heavy halofemoral traction; (2) complications with other complex intraspinal anomalies, including tethered cord, tumours, etc.; (3) short and sharp angular kyphoscoliosis, which required a osteotomy; (4) the patients that underwent any types of prophylactic neurosurgical intervention of SCM before.

\section{Preoperative traction}

All patients underwent continuous preoperative heavy halo-femoral traction. The initial traction force applied from halo was $2 \mathrm{~kg}$ to the head and $2 \mathrm{~kg}$ through distal femur traction to their lower extremity. Every day, $2 \mathrm{~kg}$ traction force increased to the head and extremity respectively, if patients well tolerated. The maximum traction force could be 33 to $50 \%$ of the whole-body weight depending on patient's tolerance. Neurological function was observed carefully during traction. The traction was applied for $18-20 \mathrm{~h}$ per day. The length of the traction period was mainly determined by the radiographic evidence of curve improvement on weekly radiographs. Traction continued till there was no significant improvement in Cobb angle on weekly radiographs $[6,7]$.

\section{Operative procedure}

During the operation, heavy halo-femoral traction was maintained (Fig. 1), and somatosensory evoked potential (SEP) and motor evoked potential (MEP) were thoroughly utilized to monitor the spinal cord functions. After exposure of posterior spinal components through a midline incision, pedicle screws (or hooks) were placed in the key vertebrae's and adjacent to them for providing multiple anchor points. Facet joint capsules, intertransverse ligaments and contracture soft tissues at the rigid segments were released completely. Then, distraction, compression, rod rotation, and derotation should be adopted for correction. All structural curves need to be fixed and fused. Allogenous or autogenous bone grafts could be implanted for fusion [8-10].

\section{Postoperative procedure}

After operation, the neurological examination was performed. Postoperatively, the patients were mobilized 


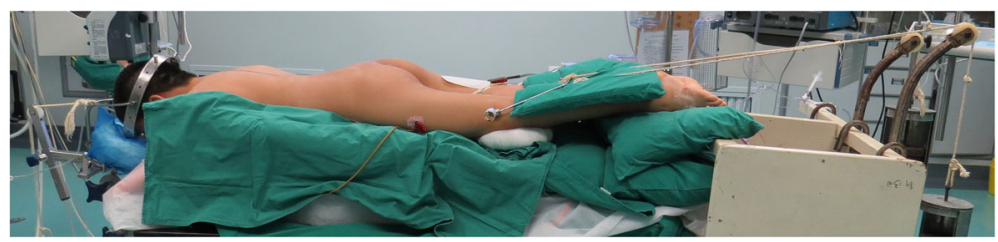

Fig. 1 During the operation, heavy halo-femoral traction was maintained

early and began to exercise 12 days later while wearing braces. All patients wore braces for an average of six months, and then gradually detached the braces.

\section{Scoliosis Research Society (SRS)-22 and modified Japanese Orthopaedic association ( $\mathrm{mJOA}$ ) scores}

SRS-22 and mJOA scores were assessed preoperatively and at the final follow up to analyse clinical outcomes and neurological function. The SRS-22 questionnaire mainly included mental health, self-image, pain, and functional activities. Furthermore, bladder function, daily activities, clinical symptoms, and subjective symptoms were assessed by the mJOA score.

\section{Evaluation of radiography and statistical analysis}

At the preoperative, postoperative, and final follow-up stages, the parameters of radiographs including coronal Cobb angle, flexibility ([preoperative Cobb angle - Cobb angle on the bending radiograph of the convex side]/preoperative Cobb angle), coronal Cobb angle after preoperative traction, correction rate were measured. The data was shown as means \pm SD and analysed using SPSS 22.0. Paired t-test was used to compare the parameters preoperatively, postoperatively and at the final follow up. $P<0.05$ indicates statistically significant difference.

\section{Results}

The mean duration of surgery was $327.08 \pm 43.99 \mathrm{~min}$ (range, 240-380 min) and the mean blood loss was $1303.33 \pm 526.86 \mathrm{ml}$ (range, $640-2100 \mathrm{ml}$ ). Thorough neurological examination was carried out in all of the patients after operation and at final follow up. No serious complications such as large vessel injury, spinal cord injury, or nerve injury occurred during operation. Moreover, there were no cases of cerebrospinal fluid leakage, death or deep infection, and none of the cases showed new irreversible neural injury.

The average period of follow up was $20.75 \pm 8.29$ months (range, 12-36 months). No complications related to instrumentation failure occurred. In one patient, the incisures of instrumentation were at the position higher than normal, which resulted in local pain and skin compression because of thinness.

\section{Correction}

The preoperative mean coronal Cobb angle was $80.38^{\circ} \pm$ $13.55^{\circ}$ (range, $60^{\circ}-113^{\circ}$ ); on the bending radiograph of the convex side, the mean Cobb angle was $68.91^{\circ} \pm$ $15.48^{\circ}$ (range, $44.5^{\circ}-98^{\circ}$ ); the mean flexibility was $15.04 \pm 7.11 \%$ (range, 5.85-28.66\%). After heavy halofemoral traction, the mean coronal Cobb angle was reduced to $56.89^{\circ} \pm 13.39^{\circ}$ (range, $35.6^{\circ}-87.5^{\circ}$ ), which showed statistically significant difference between the preoperative and post-traction data $(P<0.05)$. After posterior-only surgical correction, the postoperative mean coronal $\mathrm{Cobb}$ angle was further reduced to $32.54^{\circ} \pm 11.33^{\circ}$ (range, $19.1^{\circ}-56.2^{\circ}$ ), which showed statistically significant difference between the post-traction and postoperative data $(P<0.05)$. The postoperative mean correction rate was $60.51 \pm 7.79 \%$ (range, 48.19$69.40 \%)$. The coronal Cobb angle was $35.03^{\circ} \pm 11.22^{\circ}$ (range, $19.5^{\circ}-57.1^{\circ}$ ) at the final follow up, which revealed statistically significant difference between preoperative and the final follow-up data $(P<0.05)$, while the corrective loss rate of Cobb angle was only $3.17 \%$. The patient's trunk balance and body figure showed good improvement (Fig. 2) (Table 1).

\section{SRS-22 score}

SRS-22 scores were assessed preoperatively and at the final follow up. The mental health, self-image, pain, and functional activities scores revealed good improvement at the final follow up (Table 2), especially the mental health and self-image scores $(p<0.05)$. At the final follow up, the total score increased from $67.22 \pm 5.54$ to $84.57 \pm 4.71$. It revealed significant statistical differences between final follow-up and preoperative scores $(p<$ $0.01)$.

\section{mJOA score}

Neurological function was evaluated by mJOA score. There were no significant differences between final follow-up and preoperative scores $(p>0.05)$. At the final follow up, the total score increased from $26 \pm 2.2$ to $27 \pm 1.9$. Bladder function, daily activities, clinical symptoms, and subjective symptoms showed no significant differences between final follow-up and preoperative evaluation $(p=0.669, p=0.496, p=0.942, p=0.067$; Table 3). 


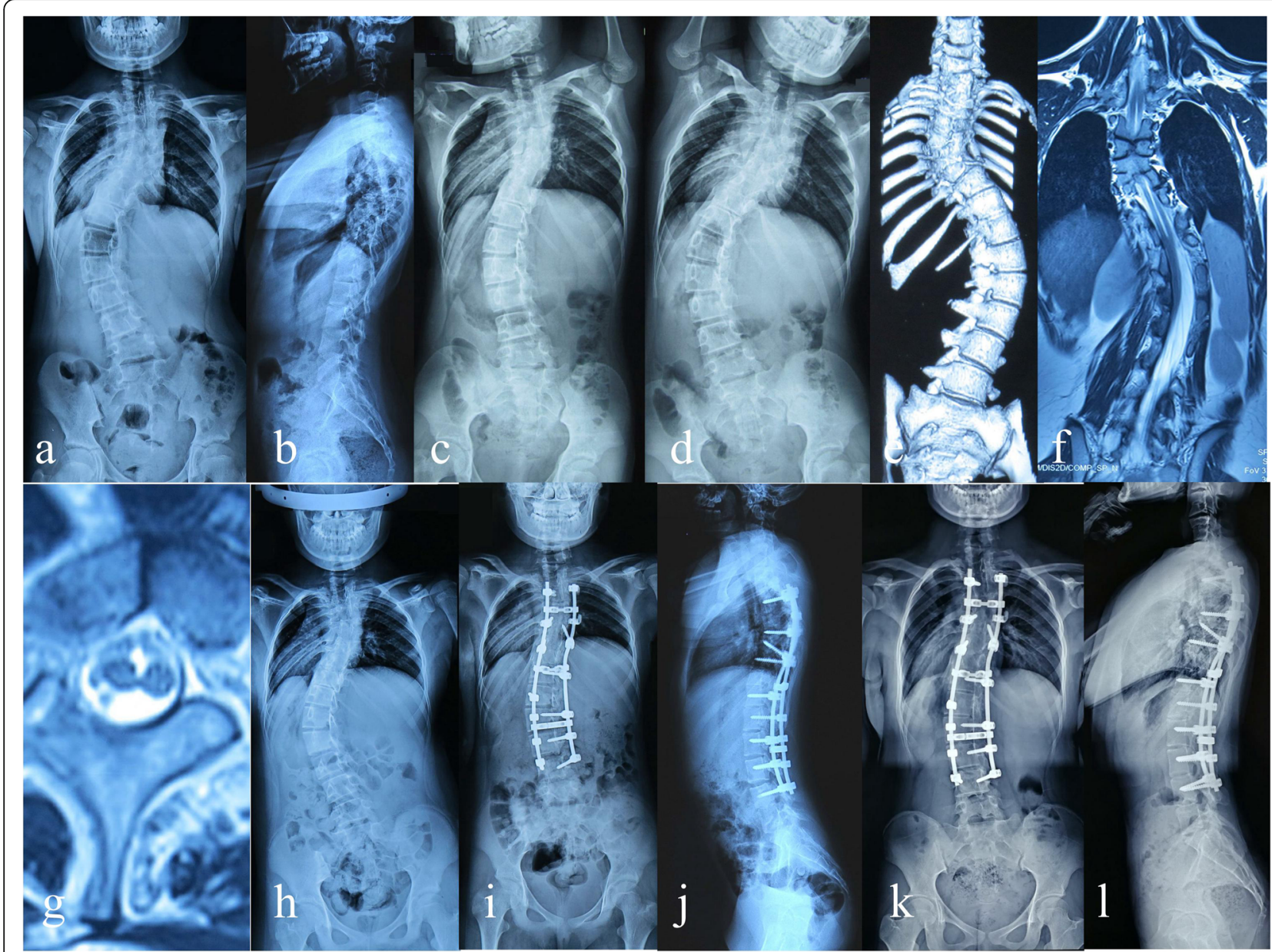

Fig. 2 A patient with RCS accompanied by SCM. a-b Preoperative radiographs show that coronal Cobb angle was $62^{\circ}$.c-d Preoperative bending radiograph of the convex side shows that Cobb angle was $45.6^{\circ}$ and flexibility was $26.45 \%$.e Preoperative CT indicates mixed defects including failure of segmentation and formation. $\mathbf{f}-\mathbf{g}$ Preoperative MRI indicates SCM type 2. $\mathbf{h}$ After heavy halo-femoral traction, the coronal Cobb angle was reduced to $38.2^{\circ}$. $\mathbf{i}$-j After posterior-only surgical correction, postoperative radiographs show that coronal Cobb angle was $21.4^{\circ}$, and correction rate was $65.48 \%$. k-I postoperative radiographs at 36 months after surgery show that coronal Cobb angle was $22.4^{\circ}$, and no signs of neurological impairment were found at the final follow-up stage

\section{Discussion}

Somites, which vertebrae derived from, enclosed neural tube during embryonic development. Any injury resulting in vertebral deformity during embryonic development may cause neural tube defects. Therefore, CS is usually accompanied by intraspinal anomalies, including SCM, tethered cord, syringomyelia, Arnold Chiari malformation, etc. There may also be multiple intraspinal anomalies at the same time $[11,12]$. In fact, CS and SCM usually occurred simultaneously in clinical practice. According to report, SCM was observed in 4.0 to $9.0 \%$ of patients with CS. The dorsolumbar and lumbar regions are the most common sites. The clinical symptoms could be summarized as following characteristics: lower extremity weakness, atrophy, and deformity, scoliosis, spinal bifida, skin lesions, sphincter dysfunction $[13,14]$.
However, most of outpatients with CS accompanied by SCM showed no signs of neurological impairment. Patients often presented with spinal deformity during their first visit to the doctor, and SCM was discovered only by chance in the examination of CT and MRI. Furthermore, the spinal cord and Dural sac may be compressed by a bony or fibrocartilaginous spur of SCM during orthopaedic surgery for CS associated with SCM, which causes neurologic injury postoperatively. The presence of SCM greatly increases the risks of correction in patients with CS.

Regarding CS accompanied by SCM, the main purpose of surgery is not only to correct spinal deformity and prevent progression of deformity, but also to prevent nerve injury. Thus far, the neurosurgical management of a bony or fibrocartilaginous spur of SCM before undertaking corrective surgery and corrective procedures are 


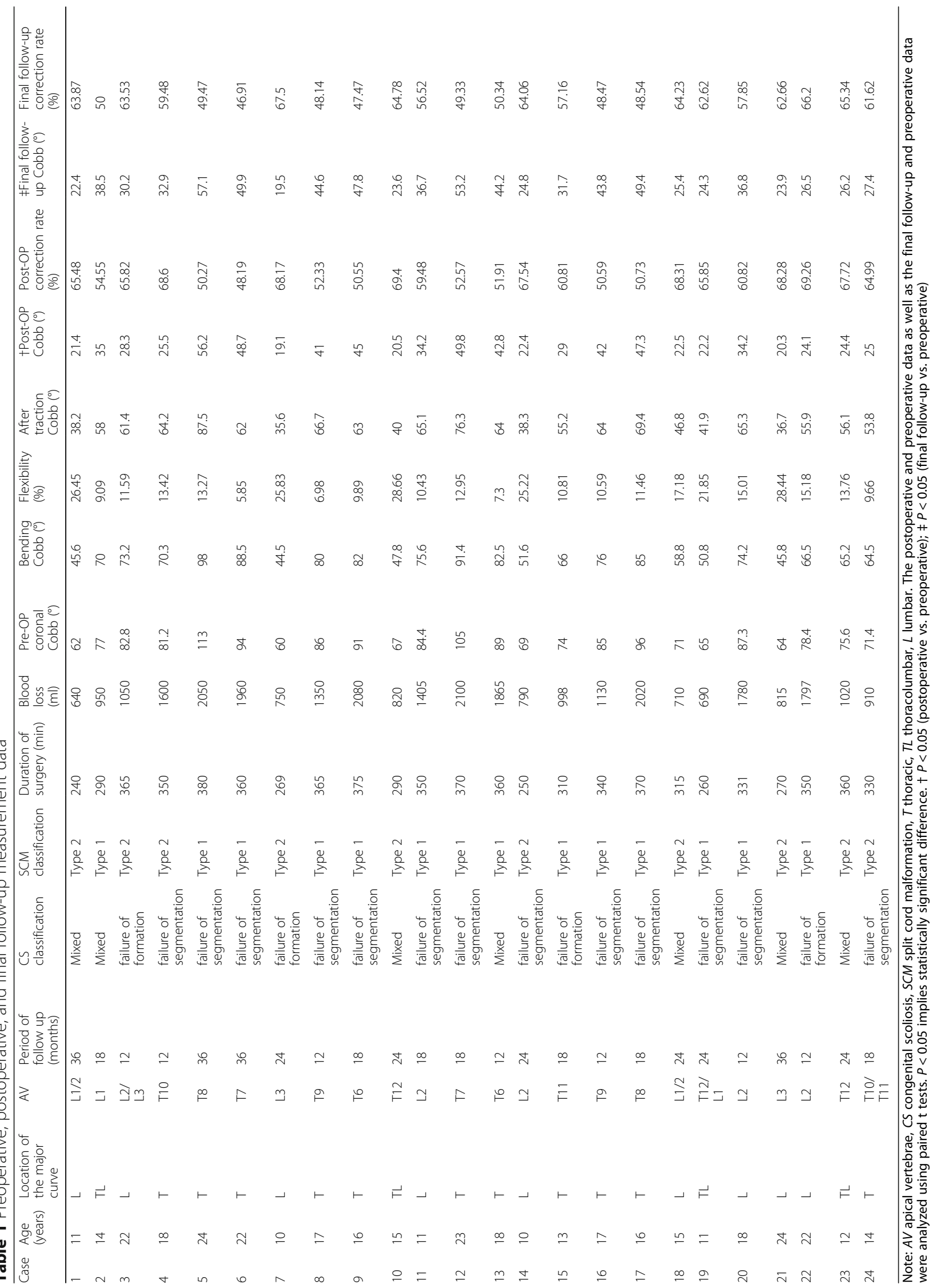


Table 2 SRS-22 score of preoperative and final follow up

\begin{tabular}{lllll}
\hline Parameters & Preoperative & Final follow-up & T value & $P$ value \\
\hline Functional activity & $17.60 \pm 2.46$ & $20.68 \pm 1.53$ & -5.208 & 0.000 \\
Pain & $20.75 \pm 1.94$ & $21.98 \pm 1.86$ & -2.240 & 0.007 \\
Self image & $14.77 \pm 2.48$ & $19.16 \pm 2.28$ & -6.384 & 0.000 \\
Mental health & $15.06 \pm 2.15$ & $19.16 \pm 1.92$ & -6.368 & 0.000 \\
OP Satisfaction & - & $7.94 \pm 1.34$ & - & - \\
SRS-22 total score & $67.22 \pm 5.54$ & $84.57 \pm 4.71$ & -11.689 & 0.000
\end{tabular}

(Note: SRS-22 questionnaires including five aspects: 1 . Recovery of functional activities of patients include question $5,9,12,15,18 ; 2$. Improvement of pain of the patients include question $1,2,8,11,17 ; 3$. Assessment of self image of the patients include question 4, 6, 2, 14, 19; 4. Assessment of mental health of the patients include question $3,7,13,16,20 ; 5$. Operation satisfaction was only answered by patients performed operation include question 21, 22)

still controversial. Ayvaz et al. [15] advised that neurosurgical interventions (spur excision and dural reconstruction) should be recommended even for neurologically asymptomatic SCM type 1 before the corrective surgery to the CS, whereas patients with SCM type 2 can be treated safely without a need of neurosurgical intervention. In SCM Type 2, it allows the spinal cord to move relatively independently in the spinal canal, because two hemi cords exist in one dural canal without substantial spur. Therefore, there is no need for additional canal work.

Some authors $[16,17]$ advocated that the approach for management of CS associated with SCM was first to perform surgery for SCM and then to perform orthopaedic surgery for correction of the spinal deformity, approximately 3 to 6 months later. The aim was to prevent spinal cord injury during deformity correction and reduce the incidence of postoperative complications of the neural system. However, there are several disadvantages of staged procedures: (1) Due to less clear anatomic landmarks, surgically complex exposure, and more blood loss, the follow-up correction becomes more difficult and complicated. In addition, at the surgical site, a preformed adhesion and possible retethering could make complex reconstructive operations such as osteotomy more difficult. (2) The patients suffer from the risks of anaesthesia and surgery more than once. (3) The staged procedures increase the financial and psychological

Table 3 mJOA score of preoperative and final follow up

\begin{tabular}{lllll}
\hline Parameters & Preoperative & Final follow-up & T value & $P$ value \\
\hline Subjective symptom & $8.1 \pm 1.1$ & $9.0 \pm 0.6$ & -1.980 & 0.067 \\
Clinical symptom & $6.3 \pm 0.2$ & $6.3 \pm 0.4$ & 0.242 & 0.942 \\
Daily activities & $12.9 \pm 1.0$ & $13.1 \pm 0.9$ & -0.686 & 0.496 \\
Bladder function & $-0.4 \pm 0.9$ & $-0.5 \pm 0.7$ & 0.430 & 0.669 \\
mJOA total score & $26 \pm 2.2$ & $27 \pm 1.9$ & -1.685 & 0.099 \\
\hline
\end{tabular}

Note: Total mJOA-score was 29 including subjective symptom from 0 to 9 score, clinical symptom from 0 to 6 score, daily activities from 0 to 14 score and bladder function from - 6 to 0 score burden in patients, and prolong hospitalization and rehabilitation.

In recent years, Hui et al. [18] have reported one-stage operation was effective and safe for the treatment of CS and SCM, but resection of bony spur was still recommended. In these cases, the correction rate of main curve was $54.5 \%$ without increasing complications. However, neurosurgical intervention itself is characterized with high risk of operation and neurological complications. For surgical interventions of SCM alone, the risk of infection, cerebrospinal fluid leakage, and neurological deterioration after neurological intervention was approximately 7 to $31 \%[14,19]$. Therefore, Feng et al. [5] compared the results of two surgical strategies for the treatment of SCM type 1 and CS. In the resection of bony spur (BR) group, the neurological complications, blood loss, and duration of surgery were significantly higher than those in the nonresection of bony spur (NR) group. Moreover, prophylactic neurosurgical intervention before corrective surgery was probably not necessary in patients with stable or intact neurological function.

Posterior spine-shortening osteotomy has recently been developed [3, 4]. The correction of the scoliosis is performed following the osteotomy. The scoliosis is corrected later by shortening and compression in the vertebrectomy gap, which relieves the longitudinal tension. Theoretically, it should prevent the spinal cord from stretch injury. However, the correction of the scoliosis in the presence of the spinal cord being tensioned by the bony or fibrocartilaginous spur in SCM still poses significant risks. Therefore, one-stage operation including resection of bony spur and subsequent spine-shortening osteotomy was recommended for preventing spurrelated complications. Nevertheless, this method presented new challenges, such as more frequent neurological complications, high level of technical requirements, difficult operation, blood loss, and operation time at a single operation. Furthermore, neurosurgical interventions (spur excision and Dural reconstruction) were still performed at the same time, and there were neurosurgical complications related to spur.

24 patients, suffered from RCS associated with SCM, were treated at our Department. No apparent neurologic dysfunction was found in all patients. All patients underwent continuous preoperative heavy halo-femoral traction, with gradual initial traction monitoring the neurological function carefully; lengthening the spine step by step. During the surgery, facet joint capsules and contracture soft tissues were released completely and widely without spur excision. After posterior-only surgical correction, the postoperative mean correction rate was $60.51 \%$. The patients' body figure and trunk balance 
showed good improvement. This correction rate was higher than that of spine-shortening osteotomy reported by some authors $[3,18]$, and was similar to that of Chen's osteotomy [4]. However, the operation time, blood loss, difficulty of operation and incidence of neurological complications were significantly lower than those reported in the literature $[4,18]$.

Therefore, our findings can be summarized in the following characteristics: (1) With initial skeletal traction gradually, the preoperative traction may increase the tolerance of spinal cord to stretch trees and ischemia from correction of curve. The patient's neurological status was frequently checked and assessed on preoperative bending and suspension position, and heavy halo-femoral traction, so as to provide a basis for intraoperative orthopaedic procedures, and diminish risks of neurological complications. (2) The preoperative heavy halo-femoral traction may significantly improve curve flexibility and spinal compliance, which allowed for a better overall correction. (3) For neurologically asymptomatic SCM, prophylactic neurosurgical intervention (spur excision and dural reconstruction) itself was characterized by increasing risk of operation and neurological complications. (4) Posterior-only surgical correction with heavy traction was performed to avoid the risks of anaesthesia and surgery more than once, and the disadvantages of spine-shortening osteotomy, such as more operation time, blood loss, difficult operation, high level of technical requirements, and frequent neurological complications. (5) The scoliosis was rigid in all the patients of this study, and the flexibility was only $15.04 \%$. During the surgery, facet joint capsules and contracture soft tissues should be released completely and widely to increase spinal flexibility. (6) Neurological monitoring (SEP and MEP) was the guarantee of the whole operation. (7) If neurological symptoms were found before surgery, or neurological symptoms occurred during heavy traction, our method was not appropriate for these patients, who needed the prophylactic neurosurgical intervention of SCM.

\section{Conclusions}

In conclusion, without prophylactic neurosurgical intervention and spine-shortening osteotomy, posterior-only surgical correction with heavy halo-femoral traction could be safe and effective for the treatment of RCS associated with SCM in decreasing the incidence of complications. However, before any procedure, appropriate surgical interventions must be chosen carefully; adequate monitoring during the surgery, preoperative heavy traction and evaluation are necessary to improve clinical results.

\section{Abbreviations}

CS: Congenital scoliosis; CT: Computed tomography; MEP: Motor evoked potential; mJOA: Modified Japanese Orthopaedic Association; MRI: Magnetic resonance imaging; RCS: Rigid congenital scoliosis; SCM: Split cord malformation; SEP: Somatosensory evoked potential; SRS: Scoliosis Research Society

\section{Acknowledgements}

Not applicable.

\section{Authors' contributions}

AD was involved in study design, data analysis and interpretation, writing the manuscript and critical revision of the manuscript. HQZ was involved in critical revision of the manuscript, statistical expertise and performing most of the analyses presented in the paper. MXT was involved in study conception and design, data collection, development of data collection instruments and critical revision of the manuscript. SHL was involved in data collection, preliminary data analysis and interpretation, critical revision of the manuscript and statistical expertise. QLG was involved in study conception and design, data analysis and interpretation, critical revision of the manuscript. YXW was involved in study conception and design, data analysis and interpretation, writing the manuscript, critical revision of the manuscript, supervision and administrative support. All authors approved the final manuscript.

\section{Funding}

The study was supported by the Natural Science Foundation of Hunan Province, China (NO. 2016JJ3160) and key research and development program of Hunan Province, China (NO. 2017SK2062). The funding bodies had no role in the design of the study or in collection, analysis, interpretation or presentation of data.

\section{Availability of data and materials}

The datasets analyzed during the current study are not publicly available because a further study about SCM is in progress in our institution, but are available from the corresponding author on reasonable request.

\section{Ethics approval and consent to participate}

The Ethics Committee in Xiangya Hospital of Central South University approved the study. All methods were performed in accordance with the relevant guidelines and regulations.

\section{Consent for publication}

Written informed consent was acquired from each of the patients (or their parents and legal guardians) to authorize treatment, imageology findings, and photographic documentation. The patients (or their parents and legal guardians) consented to the publication of their pictures as well as their anonymous and clustered data.

\section{Competing interests}

The authors declare that they have no competing interests.

Received: 30 June 2019 Accepted: 10 February 2020

Published online: 13 February 2020

References

1. Zani DD, De Zani D, Morandi N, Biggi M, Belloli AG, Riccaboni P, Rondena M, Di Giancamillo M, Pravettoni D. Imaging diagnosis--split cord malformation. Vet Radiol Ultrasound. 2010;51(1):57-60.

2. Feng F, Tan H, Li X, Chen C, Li Z, Zhang J, Shen J. Radiographic characteristics in congenital scoliosis associated with split cord malformation: a retrospective study of 266 surgical cases. BMC Musculoskelet DisordBMC Musculoskelet Disord. 2017;18(1):420.

3. Hui H, Zhang ZX, Yang TM, He BR, Hao DJ. Vertebral column resection for complex congenital kyphoscoliosis and type I split spinal cord malformation. Eur Spine JEur Spine J. 2014;23(6):1158-63.

4. Chen B, Yuan Z, Chang MS, Huang JH, Li H, Yang WZ, Luo ZJ, Tao HR. Safety and efficacy of one-stage spinal osteotomy for severe and rigid congenital scoliosis associated with Split spinal cord malformation. Spine. 2015:40(18): E1005-13. 
5. Feng F, Shen J, Zhang J, Zhao H, Zhao Y, Li Z, Xue X, Lin Y. Qiu G clinical outcomes of different surgical strategy for patients with congenital scoliosis and type I Split cord malformation. Spine. 2016;41(16):1310-6.

6. Zhang $H Q$, Wang $Y X$, Guo CF, Tang MX, Chen LQ, Liu SH, Wang YF, Chen J. Posterior-only surgery with strong halo-femoral traction for the treatment of adolescent idiopathic scoliotic curves more than $100^{\circ}$. Int Orthoplnt Orthop. 2011;35(7):1037-42.

7. Zhang HQ, Gao QL, Ge L, Wu JH, Liu JY, Guo CF, Liu SH, Lu SJ, Li JS, Yin XH, Li F. Strong halo-femoral traction with wide posterior spinal release and three dimensional spinal correction for the treatment of severe adolescent idiopathic scoliosis. Chin Med JChin Med J (Engl). 2012;125(7):1297-302.

8. Deng A, Zhang HQ, Tang MX, Liu SH, Wang YX, Gao QL. Posterior-only surgical correction of dystrophic scoliosis in 31 patients with neurofibromatosis type 1 using the multiple anchor point method. J Neurosurg Pediatr. 2017;19(1):96-101

9. Zhang HQ, Deng A, Liu SH, Chen LQ, Guo CF, Tang MX, Wu JH, Liu JY, Chen J. Adult thoracolumbar or lumbar scoliosis with Chiari malformation and syringomyelia: a retrospective study of correction and fusion strategies. Arch Orthop Trauma SurgArch Orthop Trauma Surg. 2011;131(4):475-80.

10. Zhang H, Gao Q, Wang Y, Liu S, Guo C, Tang M, Lu S, Li J. Clinical evaluation of indirect decompression treatments for degenerative adult idiopathic scoliosis. Arch Orthop Trauma SurgArch Orthop Trauma Surg. 2011;131(12): 1639-47.

11. Zhang BB, Tao HR, Wu TL, Wang L, Duan CG, Zhang T, Li T, Yang WZ, Liu M, Ma J. Safety and efficacy of single-stage surgical treatment for congenital scoliosis associated with Intraspinal mass. Sci RepSci Rep. 2017;7:41229.

12. Marks DS, Qaimkhani SA. The natural history of congenital scoliosis and kyphosis. Spine. 2009;34(17):1751-5.

13. Erşahin Y. Split cord malformation types I and II: a personal series of 131 patients. Childs Nerv SystChilds Nerv Syst. 2013;29(9):1515-26.

14. Sinha S, Agarwal D, Mahapatra AK. Split cord malformations: an experience of 203 cases. Childs Nerv SystChilds Nerv Syst. 2006;22(1):3-7.

15. Ayvaz M, Akalan N, Yazici M, Alanay A, Acaroglu RE. Is it necessary to operate all split cord malformations before corrective surgery for patients with congenital spinal deformities? Spine. 2009;34(22):2413-8.

16. Winter RB, Haven JJ, Moe JH, Lagaard SM. Diastematomyelia and congenital spine deformities. J Bone Joint Surg AmJ Bone Joint Surg Am. 1974;56(1): 27-39.

17. Liu W, Zheng D, Cui S, Zhang C, Liu Y, Jia Y, Shi T, Huang H, Hei B, Wang P. Characteristics of osseous septum of split cord malformation in patients presenting with scoliosis: a retrospective study of 48 cases. Pediatr NeurosurgPediatr Neurosurg. 2009;45(5):350-3.

18. Hui H, Tao HR, Jiang XF, Fan HB, Yan M, Luo ZJ. Safety and efficacy of 1 stage surgical treatment of congenital spinal deformity associated with split spinal cord malformation. Spine. 2012;37(25):2104-13.

19. Ayvaz M, Alanay A, Yazici M, Acaroglu E, Akalan N, Aksoy C. Safety and efficacy of posterior instrumentation for patients with congenital scoliosis and spinal dysraphism. J Pediatr OrthopJ Pediatr Orthop. 2007;27(4):380-6.

\section{Publisher's Note}

Springer Nature remains neutral with regard to jurisdictional claims in published maps and institutional affiliations.

Ready to submit your research? Choose BMC and benefit from:
- fast, convenient online submission
- thorough peer review by experienced researchers in your field
- rapid publication on acceptance
- support for research data, including large and complex data types
- gold Open Access which fosters wider collaboration and increased citations
- maximum visibility for your research: over 100M website views per year
At BMC, research is always in progress.
Learn more biomedcentral.com/submissions

